\title{
ON INGENUITY AND SEDUCTION
}

\author{
P. C. Jersild \\ Stockholm, Sweden
}

There's an American science fiction movie about how a team of surgeons save a prime minister's life. To perform the operation, the physicians first undergo a mysterious shrinking process. They turn into mini-astronauts and are injected into their patient's blood stream. Then, they sail in a tiny capsule towards the diseased area, a blood clot as I recall, and operate.

Recently, I read about a technique that could transform the story line of this movie into reality. The galloping advancement of the computer field is dependent on the art of manufacturing microchips, i.e., impressing complex patterns onto extremely small surfaces. A volume of information, which only a few decades ago required an entire office building, can today be stored on a fingernail. What is fascinating now is that we are beginning to use this new micro-technology to manufacture robots.

The robots we have seen until now have been rather big, bulky contraptions welding car chassis, or riding around on caterpillar tractors measuring radioactivity levels or disarming bombs. But if it is possible to apply micro-technology successfully, a robot's size would hardly have a lower limit. If a robot becomes small enough, it could be injected into the blood stream and perform minimal, but life-supporting, surgery (for the time being, however, without a crew). New micro-technology opens up fantastic perspectives for the wealthier segments of humanity. Who knows, in the future perhaps it will be possible to produce "living robots," after we have learned to combine and direct large protein molecules, that is.

Another development in the computer field is the new potential to integrate human beings with computers. Imagine a glove filled with electronics. This glove is connected to a screen. If you put your hand in the glove and move your fingers, you can see a picture of your hand making exactly the same movements on the screen, as if you were standing in front of a mirror. Then you can put different objects on the screen, which you can manipulate via the computer glove: turn, move, plug, pinch, etc. But to know really what you are doing, you also must simulate feeling in the hand. This feedback could be obtained by equipping the electronic glove with thin threads, which guide the hand's senses. Then, not only could you see your hand pull the cork from a bottle on the screen, but you could even feel resistance and its sudden release.

Such techniques, of course, present totally new potentials for advanced prostheses. In principle, someone who has lost a hand could be equipped with a computerized hand, which, on the screen, performs and feels what the person without a hand cannot. 
Moreover, the field of surgery could combine these two technical advancements. Computer-simulated reality is not dependent on our ordinary concept of size; objects on the screen that look like flattened planets could just as well be red blood cells. Thus, the advanced surgeon could sit before a screen and gaze into a patient's insides through a seeing micro-robot, which could show some parts enlarged a million times. The doctor could guide injected robots throughout the body with sensitive computer gloves. Via satellite link, a patient in Australia could be saved painlessly by a surgeon sitting in front of a computer in Paris - with neither loss of blood nor time.

Having gone this far, I stop and ponder if I should not be politely ashamed of my delight in technology. Because is it not our creative ingenuity - a child's delight in clever things - that more than money often drives our scary technological development, the very thing that seems so impossible to control?

In the Pentagon, Silicon Valley, and other similar conclaves, thousands of adults sit and putter with microchips, genes, or star wars-men and women who look upon the world as a Christmas present just opened, a magic box or Tinker Toys, which can keep us awake throughout the forever long and happy Christmas night.

One who is hypnotized by technology has much in common with one who creates or admires art. Art, whether painting, music, or literature, is a sort of framed miniworld or colorful private universe, a closed room where one can escape and not only be entertained but also wonder or admire without being disturbed by external reality. Reality is what one, newly awakened and a bit chilly, meets when one leaves the movie theater on stiff legs.

This is how artists and scientist/technicians live in well-defined worlds. I cannot see that the artist would be morally superior to someone who invents computer software or winds their way forward through the genetic code. Creative work spellbinds all its practitioners. It is only before, when we choose what we should venture intoand afterward, when we are confronted with what we have achieved - that our possible consciousness is tried and judgment can be passed on us. 